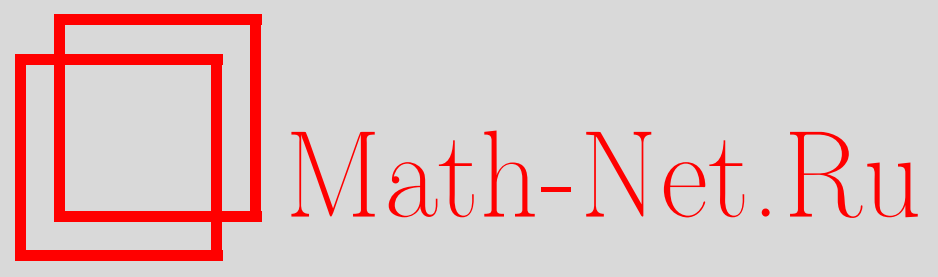

Д. А. Куропаткин, Вероятности событий, связанных с общими предками двух вершин в обобщенной модели рекурсивных деревьев, Дискрет. матем., 1999, том 11, выпуск 4, 58-64

DOI: https://doi.org/10.4213/dm399

Использование Общероссийского математического портала Math-Net.Ru подразумевает, что вы прочитали и согласны с пользовательским соглашением http://www.mathnet.ru/rus/agreement

Параметры загрузки:

IP : 54.224 .135 .184

26 апреля 2023 г., 16:11:11

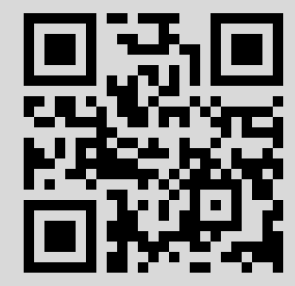




\title{
Вероятности событий, связанных с общими предками двух вершин в обобщенной модели рекурсивных деревьев
}

(C) 1999 г. Д. А. Куропаткин

\begin{abstract}
Случайное дерево $T_{n}$ с $n$ вершинами и $n-1$ ребрами называется обобщенным рекурсивным, если либо $n=1$, либо $n>1$ и $T_{n}$ получается присоединением $n$-й вершины к какой-либо вершине случайного рекурсивного дерева $T_{n-1}$. При этом вероятность выбора конкретной вершины определяется некоторой последовательностью $\left\{\alpha_{i}: \alpha_{i}>0\right\}_{i=1}^{\infty}$. В настоящей работе исследуются вероятности некоторых событий, касающихся общих предков вершин с произвольными номерами.
\end{abstract}

\section{1. Введение}

Рекурсивные деревья являются одним из интересных и хорошо изученных классов помеченных деревьев (см., например, [1-3]). В работе [1] была предложена модель случайных рекурсивных деревьев, которая обобщает ранее известные конструкции. Построение интересующих нас случайных деревьев $T_{n}$ с $n$ вершинами, $n \geqslant 1$, производится по последоватєльности

$$
\left\{\alpha_{i}\right\}_{i=1}^{\infty} \quad \alpha_{1}, \alpha_{2}, \ldots, \alpha_{l}>0, \quad \alpha_{i} \geqslant 0, \quad i>l,
$$

следующим образом. При $n=1$ дерево $T_{n}$ состоит из изолированной вершины (корня) с меткой 1 . Пусть теперь $n>1$ и дерево $T_{n-1}$ построено. Положим

$$
S_{k}=\sum_{i=1}^{k} \alpha_{i}, \quad k \geqslant 1, \quad P_{n}^{k}=\frac{\alpha_{k}}{S_{n-1}} \quad k=1,2, \ldots, n-1
$$

Выберем из множества $\{1,2, \ldots, n-1\}$ случайно некоторое число $\xi$ с законом распределения $\mathbf{P}\{\xi=k\}=P_{n}^{k}$ и соединим ребром вершину $n$ с вершиной $\xi$. Полученное таким образом дерево и будет интересующим нас случайным рекурсивным деревом $T_{n}$ с $n$ вершинами.

Если все $\alpha_{i}$ равны одной и той же постоянной, то мы приходим к обычной (равномерной) модели рекурсивных деревьев. Примеры использования случайных рекурсивных деревьев можно найти в $[2,3]$. 
Для любых $1 \leqslant k<n$ определим случайные величины $J_{k}^{n}$ следующим образом:

$$
J_{k}^{n}= \begin{cases}1, & \text { если вершина } k \text { лежит на пути из корня в вершину } n, \\ 0 & \text { в противном случае. }\end{cases}
$$

Положим для простоты

$$
P_{k}(n, m)=\mathbf{P}\left\{J_{k}^{n} J_{k}^{m}=1\right\}, \quad 1 \leqslant k<m<n .
$$

Заметим, что в работе [1] была сделана попытка найти вероятность $P_{k}(n, m)$, но в рассуждения вкралась ошибка. Укажем на нее.

Будем использовать обозначения из [1]. Под $T_{m-1}^{k}$ понимается поддерево $T_{m-1}$, корнем которого является вершина $k$. Пусть

$$
\alpha\left(T_{m-1}^{k}\right)=\sum_{\nu_{i} \in T_{m-1}^{k}} \alpha_{i}
$$

где $\nu_{i}$ - вершина с номером $i$. В [1] при нахождении $P_{k}(n, m)$ используется равенство

$$
\mathbf{E}\left[\alpha\left(T_{m}^{k}\right) \mid J_{k}^{m}=1\right]=\alpha_{m}+\mathbf{E} \alpha\left(T_{m-1}^{k}\right),
$$

которое не поясняется и не доказывается. Покажем на примере, что оно не верно.

Пример 1. Пусть $\alpha_{i}=1$ для любоио $i \in \mathbf{N}, k-$ произвольное целое положительное число, $m=k+2$. Тогда

$$
\begin{aligned}
\mathbf{E}\left[\alpha\left(T_{m}^{k}\right) \mid J_{k}^{m}=1\right] & =\sum_{x} x \mathbf{P}\left\{\alpha\left(T_{m}^{k}\right)=x \mid J_{k}^{m}=1\right\} \\
& =\sum_{x} \frac{x \mathbf{P}\left\{\alpha\left(T_{m}^{k}\right)=x, J_{k}^{m}=1\right\}}{\mathbf{P}\left\{J_{k}^{m}=1\right\}}
\end{aligned}
$$

где суммирование ведется по всем значениям $x$, которые принимает $\alpha\left(T_{m}^{k}\right)$.

В нашем случае из (2) с учетом соотношения

$$
\mathbf{P}\left\{J_{k}^{m}=1\right\}=\frac{\alpha_{k}}{S_{k}}, \quad k=1,2, \ldots, m
$$

(см. [1]), следует, что

$$
\mathbf{E}\left[\alpha\left(T_{k+2}^{k}\right) \mid J_{k}^{k+2}=1\right]=3 \frac{2}{k(k+1)} k+2 \frac{k-1}{k(k+1)} k=\frac{2(k+2)}{k+1} .
$$

Применяя аналогичные рассуждения, находим, что

$$
1+\mathbf{E} \alpha\left(T_{k+1}^{k}\right)=1+\sum_{y} y \mathbf{P}\left\{\alpha\left(T_{k+1}^{k}\right)=y\right\}=1+\frac{2}{k}+\frac{k-1}{k}=\frac{2 k+1}{k} .
$$

Однако

$$
\frac{2(k+2)}{k+1} \neq \frac{2 k+1}{k}
$$

что опровергает (1). 


\section{2. Нахождение $P_{k}(n, m)$}

В данном разделе мы исправляем неточность, допущенную в [1], и доказываем следующее утверждение.

Теорема 1. Для любых $1 \leqslant k<m<n$

$$
P_{k}(n, m)=\frac{\alpha_{k}}{S_{k}}\left(1-\frac{S_{k-1}}{S_{k+1}} \prod_{j=k+2}^{m}\left(1-\frac{\alpha_{j}-\alpha_{j-1}}{S_{j}}\right)\right) .
$$

Замечание 1. Ясно, что если $\alpha_{j}$ есть одна и та же постоянная для всех $j, j \in$ $\{k+2, \ldots, m\}$, то

$$
P_{k}(n, m)=\frac{\alpha_{k}}{S_{k}} \frac{\alpha_{k+1}+\alpha_{k}}{S_{k+1}}
$$

Если $\alpha_{j}$ есть одна и та же постоянная для всех $j \in \mathbf{N}$, то

$$
P_{k}(n, m)=\frac{2}{k(k+1)}
$$

Вероятность $P_{k}(n, m)$ не зависит от параметра $n$.

Прежде чем перейти к доказательству теоремы, докажем несколько вспомогательных утверждений.

Лемма 1. Для $1 \leqslant k<m<n$

$$
P_{k}(n, m)=\sum_{i=m+1}^{n-1} P_{k}(i, m) \frac{\alpha_{i}}{S_{n-1}}+\frac{\alpha_{m}}{S_{n-1}} \frac{\alpha_{k}}{S_{k}}+\sum_{i=k+1}^{m-1} P_{k}(m, i) \frac{\alpha_{i}}{S_{n-1}}+\frac{\alpha_{k}}{S_{n-1}} \frac{\alpha_{k}}{S_{k}} .
$$

Доказательство. Назовем вершину $i$ ближайшим предком вершины $n$, если вершина $n$ непосредственно присоединяется к вершине $i$. Равенство (4) является формулой полной вероятности, где сумма берется по всем возможным ближайшим предкам вершины $n$. Если $i-$ ближайший предок вершины $n$, то искомая вероятность равна произведению

$$
\mathbf{P}\{k \text { лежит на пути из } m \text { в } i\} P_{n}^{i} \text {. }
$$

Тогда ясно, что в правой части равенства (4) первая сумма соответствует значениям $i \in\{m+1, \ldots, n-1\}$, следующее слагаемое соответствует $i=m$, вторая сумма соответствует $i \in\{k+1, \ldots, m-1\}$ и, наконец, последнее слагаемое получается при $i=k$. Лемма доказана.

Покажем теперь, что $P_{k}(n, m)$ не зависит от первого параметра. Обозначим для краткости $P_{k}(m+1, m)$ через $P_{k}(m)$.

Лемма 2. Для любых $1 \leqslant k<m<n$

$$
P_{k}(n, m)=P_{k}(m)
$$


Доказательство. Используя (4), находим, что

$$
\begin{aligned}
P_{k}(n+1, m) & =\frac{1}{S_{n}}\left(P_{k}(n, m) \alpha_{n}+\sum_{i=m+1}^{n-1} P_{k}(i, m) \alpha_{i}+\frac{\alpha_{m} \alpha_{k}}{S_{k}}+\sum_{i=k+1}^{m-1} P_{k}(m, i) \alpha_{i}+\frac{\alpha_{k}^{2}}{S_{k}}\right) \\
& =\frac{1}{S_{n}}\left(P_{k}(n, m) \alpha_{n}+S_{n-1} P_{k}(n, m)\right)=P_{k}(n, m) .
\end{aligned}
$$

Отсюда немедленно получаем (5). Лемма доказана.

Доказательство теоремъ 1. Используя (5), преобразуем равенство (4). Получаем, что

$$
\begin{aligned}
S_{n-1} P_{k}(m) & =\sum_{i=m+1}^{n-1} P_{k}(m) \alpha_{i}+\sum_{i=k+1}^{m-1} P_{k}(i) \alpha_{i}+\frac{\alpha_{k}}{S_{k}}\left(\alpha_{k}+\alpha_{m}\right) \\
S_{m} P_{k}(m) & =\sum_{i=k+1}^{m-1} P_{k}(i) \alpha_{i}+\frac{\alpha_{k}}{S_{k}}\left(\alpha_{k}+\alpha_{m}\right)
\end{aligned}
$$

Используя последнюю формулу, вероятность $P_{k}(m+1)$ можно выразить через $P_{k}(m)$ при любом $m>k \geqslant 1$ :

$$
\begin{aligned}
P_{k}(m+1) & =\frac{1}{S_{m+1}}\left(\sum_{i=k+1}^{m-1} P_{k}(i) \alpha_{i}+\frac{\alpha_{k}^{2}}{S_{k}}+P_{k}(m) \alpha_{m}+\frac{\alpha_{k} \alpha_{m+1}}{S_{k}}\right) \\
& =\frac{1}{S_{m+1}}\left(S_{m} P_{k}(m)-\frac{\alpha_{k} \alpha_{m}}{S_{k}}+P_{k}(m) \alpha_{m}+\frac{\alpha_{k} \alpha_{m+1}}{S_{k}}\right) \\
& =P_{k}(m)\left(1-\frac{\alpha_{m+1}-\alpha_{m}}{S_{m+1}}\right)+\frac{\alpha_{k}}{S_{k}} \frac{\alpha_{m+1}-\alpha_{m}}{S_{m+1}}
\end{aligned}
$$

С учетом очевидного равенства

$$
P_{k}(k+2, k+1)=\frac{\alpha_{k}}{S_{k}} \frac{\alpha_{k}+\alpha_{k+1}}{S_{k+1}}
$$

получаем, что для любого $m>k$

$$
\begin{aligned}
P_{k}(m)=\frac{\alpha_{k}}{S_{k}} \frac{\alpha_{k+1}+\alpha_{k}}{S_{k+1}} \prod_{i=k+2}^{m}\left(1-\frac{\alpha_{i}-\alpha_{i-1}}{S_{i}}\right) & \\
& \quad+\frac{\alpha_{k}}{S_{k}} \sum_{i=k+2}^{m} \frac{\alpha_{i}-\alpha_{i-1}}{S_{i}} \prod_{j=i+1}^{m}\left(1-\frac{\alpha_{j}-\alpha_{j-1}}{S_{j}}\right) .
\end{aligned}
$$

Здесь и далее считаем, что если в произведении нижний индекс больше верхнего, то произведение равно 1.

Преобразуем последнее выражение. Полагая

$$
\Delta_{i}=\prod_{j=i}^{m}\left(1-\frac{\alpha_{j}-\alpha_{j-1}}{S_{j}}\right)
$$


находим, что

$$
\Delta_{i+1}-\Delta_{i}=\frac{\alpha_{i}-\alpha_{i-1}}{S_{i}} \prod_{j=i+1}^{m}\left(1-\frac{\alpha_{j}-\alpha_{j-1}}{S_{j}}\right)
$$

Следовательно,

$$
P_{k}(m)=\frac{\alpha_{k}}{S_{k}} \frac{\alpha_{k+1}+\alpha_{k}}{S_{k+1}} \Delta_{k+2}+\left(1-\Delta_{k+2}\right) \frac{\alpha_{k}}{S_{k}}=\frac{\alpha_{k}}{S_{k}}\left(1-\frac{S_{k-1}}{S_{k+1}} \Delta_{k+2}\right) .
$$

Доказательство закончено.

\section{3. О расстоянии до ближайшего общего предка}

Пусть, как и ранее, $1 \leqslant k<m<n$. Множество вершин, которые лежат и на пути от корня к вершине $n$ и на пути от корня к вершине $m$ не пусто. Вершину из этого множества с наибольшим номером будем называть ближайшим общим предком $n$ и $m$. Вероятность того, что номер ближайшего общего предка не меньше $k$ обозначим через $\tilde{P}_{k}(n, m)$.

Теорема 2. Для любых $1 \leqslant k<m<n$

$$
\tilde{P}_{k}(n, m)=1-\prod_{j=k+2}^{m}\left(1-\frac{\alpha_{j} \alpha_{j-1}}{S_{j}}\right)\left(1-\frac{\alpha_{k+1}}{S_{k+1}}-\frac{\alpha_{k}^{2}}{S_{k} S_{k+1}}\right) .
$$

Замечание 2. Если при любом $j \in\{k+2, \ldots, m\}$ все $\alpha_{j}$ равны одной и той же постоянной, то

$$
\tilde{P}_{k}(n, m)=\frac{\alpha_{k+1}}{S_{k+1}}+\frac{\alpha_{k}^{2}}{S_{k} S_{k+1}} .
$$

Если $\alpha_{j}$ есть одна и та же постоянная при всех $j \in \mathbf{N}$, то

$$
\tilde{P}_{k}(n, m)=\frac{1}{k}
$$

Вероятность $\tilde{P}_{k}(n, m)$ не зависит от параметра $n$.

Пусть $q_{k}(m, n)$ есть вероятность того, что номер ближайшего предка вершин $m$ и $n$ равен $k$, где $k<m<n$.

Следствие 1. Для любъх $1 \leqslant k<m<n$

$$
q_{k}(m, n)=\alpha_{k}^{2} \frac{S_{k+1}+\alpha_{k+1}}{S_{k} S_{k+1} S_{k+2}} \prod_{j=k+3}^{m}\left(1-\frac{\alpha_{j}-\alpha_{j-1}}{S_{j}}\right) .
$$

Последняя формула вытекает из равенства

$$
q_{k}(m, n)=\tilde{P}_{k}(m)-\tilde{P}_{k+1}(m) .
$$

Доказывать теорему 2 будем аналогично теореме 1. Все рассуждения идейно остаются такими же, поэтому приводить их не будем, а остановимся лишь на ключевых моментах и формулировках. 
Лемма 3. Для любых $n>m>k \geqslant 1$

$$
\tilde{P}_{k}(n, m)=\sum_{i=m+1}^{n-1} \tilde{P}_{k}(i, m) \frac{\alpha_{i}}{S_{n-1}}+\frac{\alpha_{m}}{S_{n-1}}+\sum_{i=k+1}^{m-1} \tilde{P}_{k}(m, i) \frac{\alpha_{i}}{S_{n-1}}+\frac{\alpha_{k}}{S_{n-1}} \frac{\alpha_{k}}{S_{k}}
$$

Лемма 4. Для любых $n>m>k \geqslant 1$

$$
\tilde{P}_{k}(n, m)=\tilde{P}_{k}(m)
$$

əде по определению $\tilde{P}_{k}(n)$ есть $\tilde{P}_{k}(m+1, m)$.

Доказательство теоремъ 2. Так же, как в теореме 1, получаем, что

$$
\tilde{P}_{k}(m+1)=\tilde{P}_{k}(m)\left(1-\frac{\alpha_{m+1}-\alpha_{m}}{S_{m+1}}\right)+\frac{\alpha_{m+1}-\alpha_{m}}{S_{m+1}} .
$$

Учитывая, что

$$
\tilde{P}_{k}(k+2, k+1)=\frac{1}{S_{k+1}}\left(\alpha_{k+1}+\frac{\alpha_{k}^{2}}{S_{k}}\right)
$$

приходим к соотношению

$$
\begin{aligned}
\tilde{P}_{k}(m)=\frac{1}{S_{k+1}}\left(\alpha_{k+1}+\frac{\alpha_{k}^{2}}{S_{k}}\right) \prod_{j=k+2}^{m}(1 & \left.-\frac{\alpha_{j}-\alpha_{j-1}}{S_{j}}\right) \\
& +\sum_{i=k+2}^{m} \frac{\alpha_{i}-\alpha_{i-1}}{S_{i}} \prod_{j=i+1}^{m}\left(1-\frac{\alpha_{j}-\alpha_{j-1}}{S_{j}}\right) .
\end{aligned}
$$

Следовательно,

$$
\begin{aligned}
\tilde{P}_{k}(m) & =\frac{1}{S_{k+1}}\left(\alpha_{k+1}+\frac{\alpha_{k}^{2}}{S_{k}}\right) \Delta_{k+2}+1-\Delta_{k+2} \\
& =1-\Delta_{k+2}\left(1-\frac{\alpha_{k+1}}{S_{k+1}}-\frac{\alpha_{k}^{2}}{S_{k} S_{k+1}}\right) .
\end{aligned}
$$

Доказательство закончено.

\section{4. Расстояние между вершинами}

Согласно [4], расстояние $D_{m n}$ между вершинами $m$ и $n, n>m$, равно числу ребер лежащих на пути, соединяющем эти вершины. В [1] было найдено математическое ожидание $D_{m n}$, но в приведенном доказательстве существенно использовалось неверное значение $P_{k}(n, m)$. Ввиду этого теорема 3.1 из [1] ошибочна. Следующее утверждение восполняет указанный пробел.

Теорема 3. Для любих $n>m \geqslant 1$

$$
\mathbf{E} D_{m n}=\sum_{k=1}^{n-1} \frac{\alpha_{k}}{S_{k}}+\sum_{k=1}^{m-1} \frac{\alpha_{k}}{S_{k}}-2 \frac{\alpha_{m}}{S_{m}}-2 \sum_{k=2}^{m-1} \frac{\alpha_{k}}{S_{k}}\left(1-\frac{S_{k-1}}{S_{k+1}} \prod_{j=k+2}^{m}\left(1-\frac{\alpha_{j}-\alpha_{j-1}}{S_{j}}\right)\right) .
$$


Доказательство. Как и в [1], пусть

$$
D_{n}=\sum_{k=1}^{n-1} J_{k}^{n}
$$

- число ребер на пути, соединяющем корень с вершиной $n$. Обозначим через $H_{m n}$ число ребер, которые принадлежат и пути от корня до $n$, и пути от корня до $m$.

Очевидно, что

$$
D_{m n}=\left(D_{n}-H_{m n}\right)+\left(D_{m}-H_{m n}\right) .
$$

Также ясно, что

$$
H_{m n}=\sum_{k=2}^{m} J_{k}^{n} J_{k}^{m}
$$

где $J_{m}^{m} \equiv 1$. Учитывая, что

$$
\mathrm{E} D_{n}=\sum_{k=1}^{n-1} \frac{\alpha_{k}}{S_{k}}
$$

находим, что

$$
\mathbf{E} D_{m n}=\sum_{k=1}^{n-1} \frac{\alpha_{k}}{S_{k}}+\sum_{k=1}^{m-1} \frac{\alpha_{k}}{S_{k}}-2 \sum_{k=2}^{m-1} \mathrm{E} J_{k}^{n} J_{k}^{m}-2 J_{m}^{n} .
$$

Используя равенство $\mathrm{E} J_{k}^{n} J_{k}^{m}=P_{k}(n, m)$ и выражение для $P_{k}(n, m)$, получаем формулу, приведенную в формулировке теоремы. Доказательство закончено.

Если все $\alpha_{j}$ равны одной и той же постоянной при $j \in \mathbf{N}$ (что соответствует простым рекурсивным деревьям), то

$$
\mathbf{E} D_{m n}=\sum_{k=1}^{n-1} \frac{1}{k}+\sum_{k=1}^{m} \frac{1}{k}-2+\frac{1}{m} .
$$

Полученное равенство согласуется с формулой (12) из [4].

\section{Список литературы.}

1. Shapiro A., A generalized distribution model for random recursive trees. Acta Inform. (1997) 34, 211-216.

2. Gastwirth J.L., A probability model of a pyramid scheme. Amer. Statistician (1977) 31, 79-82.

3. Moon J.W., The distance between nodes in recursive trees. London Math. Soc. Lecture Notes (1974) 13, 125-132.

4. Dobrow R.P., On the distribution of distances in recursive trees. J. Appl. Prob. (1996) 33, 749-757. 